\title{
Preparation and Characterization of Large-Area and Flexible Lead Zirconate Titanate/Polyvinyl-Butyral/Additives Composite Films for Piezoelectric Sensor Application
}

\author{
Xiaoliang Fang, Jingjing He, ${ }^{*}$ and Yulong Zhang \\ School of Reliability and System Engineering, Science \& Technology Laboratory on Reliability \\ \& Environment Engineering, Beihang University, \\ XueYuan Road No.37, Haidian District, Beijing 100191, China \\ (Received April 17, 2015; accepted March 18, 2016)
}

Keywords: piezoelectric composite film, $\mathrm{PZT} / \mathrm{PVB} /$ additives, piezoelectric sensor, SHM

To satisfy the requirement of structural health monitoring (SHM) using light sensors with high sensitivity, novel large-area and flexible lead zirconate titanate (PZT)/polyvinyl-butyral (PVB)/ additives composite films were fabricated by the tape casting method. The microstructure and the piezoelectric and dielectric properties of the PZT/PVB/additives composite films were investigated systematically. Owing to the additives (dibutyl phthalate + castor oil), the flexibility and uniformity of the PZT/PVB composite films were improved significantly compared with conventional PZT/ PVB composite films. Moreover, the surfaces of the PZT/PVB/additives composite films are smooth and highly resistant to cracking. Scanning electron microscopy (SEM) was adopted to analyze the microstructure of the films. The microstructure analysis shows that microholes exist in the PZT/PVB composite films and an agglomeration phenomenon is noticeable. In contrast, PZT particles are well dispersed in the PVB matrix and there are few obvious microholes in the $\mathrm{PZT} / \mathrm{PVB} /$ additives composite films. Furthermore, from energy-dispersive spectroscopy (EDS) results, it can be concluded that PZT particles were deposited at the bottom surface of the PZT/ PVB composite films. However, uniform deposition of PZT particles is achieved in the PZT/PVB/ additives composite films. In addition, owing to the additives, the improvement of the piezoelectric and dielectric properties of $\mathrm{PZT} / \mathrm{PVB} /$ additives composite films is observed at a high volume fraction of $\operatorname{PZT~}[\varphi(\mathrm{PZT})>60 \%]$. At $\varphi(\mathrm{PZT})=85 \%$, the largest piezoelectric constant $\left(d_{33}\right)$ reaches $44 \mathrm{pC} / \mathrm{N}$, which is much higher than that of the reported PZT/PVDF films and PZT/PVB films. Moreover, the dielectric constant $\left(\varepsilon_{\mathrm{r}}\right)$ of the PZT/PVB/additives composite films (at room temperature and a frequency of $1 \mathrm{kHz}$ ) is higher than that of the PZT/PVB composite films with the same $\varphi(\mathrm{PZT})$, and the dielectric loss $(\tan \delta)$ is lower. Both the improved piezoelectric and dielectric properties are desirable for new piezoelectric sensor applications.

\section{Introduction}

Lead zirconate titanate (PZT) is a solid solution of lead zirconate and lead titanate. Owing to its excellent piezoelectric and dielectric characteristics, PZT is widely used for sensors and actuators. $^{(1-3)}$ PZT is a stiff and brittle ceramic material with a relatively high density. Therefore,

"Corresponding author: e-mail: hejingjing@buaa.edu.cn 
sensors fabricated using PZT are difficult to attach to curved surfaces to form a sensor array. Additionally, conventional PZT sensors are not suitable for use in aircraft structures, where the extra weight introduced by PZT sensors is a concern. ${ }^{(4,5)}$ Polymers have many advantages, such as high flexibility and low density. In particular, polymers can be fabricated to form large films, but polymers have unsatisfactory piezoelectric and dielectric properties. ${ }^{(4)}$

PZT/polymer composites have both the piezoelectric and dielectric properties of PZT and the mechanical properties of polymers. ${ }^{(6,7)}$ In recent years, studies on PZT/polymer composites have drawn increasing attention. Polyvinylidene fluoride (PVDF) is chosen as a polymer matrix owing to its good flexibility and piezoelectric properties as well as low stiffness and mechanical impedance. Many studies focused on improving the piezoelectric and dielectric properties of PZT/PVDF composites. ${ }^{(8-13)}$ Ideally, a larger volume fraction of PZT corresponds to improved piezoelectric and dielectric properties; however, when the volume fraction of PZT is larger than $70 \%$, the flexibility and strength of the composites can be greatly reduced. ${ }^{(14,15)}$ Furthermore, the PZT particles may aggregate and be deposited in large numbers at a high volume fraction of PZT, decreasing the piezoelectric and dielectric performance of the composites. ${ }^{(16)}$ Therefore, simultaneously improving the flexibility and the piezoelectric and dielectric properties of PZT/polymer composites remains a major challenge for realizing their applications.

Polyvinyl-butyral (PVB) films exhibit high material strength due to excellent cohesiveness, and have drawn great attention in the development of PZT/PVB composite films (PPFs). ${ }^{(17-19)}$ Dong et al. prepared a PPF with a diameter of $12 \mathrm{~mm}$ and a thickness of $1.2 \mathrm{~mm}$ by the hot pressing method, and demonstrated that composite films with a high volume fraction of PZT (85\%) can be fabricated. ${ }^{(17)}$ However, composite films prepared by the hot pressing method are usually small and thick, resulting in unsatisfactory flexibility and microstructural nonuniformity. This potential disadvantage limits their applications in, for example, micro-electromechanical systems (MEMS) ${ }^{(2)}$ and the structural health monitoring (SHM) of aircraft structures. ${ }^{(20)}$

Current practice suggests adding dibutyl phthalate (DBP) to a polymer as a plasticizer to make the polymer more flexible. ${ }^{(21,22)}$ In addition, introducing castor oil can produce a more homogeneous distribution of ceramic particles in the polymer matrix. ${ }^{(23)}$ A novel composite film, namely a PZT/ $\mathrm{PVB} /$ additives composite film (PPAF), was prepared by the tape casting method in this study to further improve the flexibility and piezoelectric and dielectric properties of a PPF. Using the tape casting method allows us to produce larger and thinner composite films. ${ }^{(24)}$ During the fabrication process, additives (DBP and castor oil) were introduced to enhance the flexibility and uniformity of the PPAF. The effects of the additives on the structural, dielectric, and piezoelectric properties of PPAFs were investigated. Experimental results indicate that the PPAFs made by the proposed method exhibit improved flexibility and dielectric and piezoelectric properties compared with conventional PPFs.

\section{Materials and Methods}

The PZT powder (P-43) was produced by Zibo Yuhai Electronic Ceramics Co., Ltd. The piezoelectric strain constant $d_{33}$ and the relative dielectric constant $\varepsilon_{\mathrm{r}}$ are $300 \mathrm{pC} / \mathrm{N}$ and 1500 , respectively. The particle size of the PZT powder is within the range of $2-5 \mu \mathrm{m}$. The PVB powder and DBP liquid were supplied by Yingkou Tianyuan Chemical Industry Institute. The castor oil was provided by Shanghai Beite Chemical Co., Ltd. 
Ethanol and butanone were mixed together at a volume ratio of 47:53 to prepare an organic solvent. The commercial PVB powder, DBP liquid, and castor oil were dissolved uniformly in the organic solvent at a volume ratio of 6.9:2.6:1. The PZT powder with a density of $7.5 \mathrm{~g} / \mathrm{cm}^{3}$, which is much higher than that of the PVB powder $\left(1.09 \mathrm{~g} / \mathrm{cm}^{3}\right)$, was finally added to the organic solvent. To obtain a suitable viscosity of the solution, $100 \mathrm{~g}$ PZT powder should be mixed with $120 \mathrm{ml}$ organic solvent. To investigate the effect of additives on the properties of the composite films with different PZT volume ratios, several composite films were fabricated at volume ratios of PZT to PVB of 50:50, 60:40, 70:30, 80:20, and 85:15.

The above solution was stirred in a ball mill for $5 \mathrm{~h}$ at $80 \mathrm{rpm}$ to uniformly distribute the PZT powder in the solution. After that, the solution was placed into a vacuum chamber for $30 \mathrm{~min}$ to drive the air bubbles out of the solution. Finally, the solution was poured into a tape casting machine. The height of the blade was set as $0.2 \mathrm{~mm}$ and the speed was set as $0.3 \mathrm{~mm} / \mathrm{s}$. A 70-90- $\mu$ m-thick PZT/PVB/additives (DBP + castor oil) composite film was finally obtained after drying for $3 \mathrm{~h}$ at $90{ }^{\circ} \mathrm{C}$. A photograph of the composite film is shown in Fig. 1. For the sake of comparative analysis, PPFs with different volume fractions of PZT were also prepared by the same method.

Gold electrodes were sputtered on both sides of the composite film in vacuum by the ion sputtering method. The area of each electrode was $10 \times 10 \mathrm{~mm}^{2}$. After that, the polarization of the composite film was performed along the thickness direction in a silicone oil bath. The polarization voltage was $12 \mathrm{kV} / \mathrm{mm}$, the temperature during polarization was maintained at $110{ }^{\circ} \mathrm{C}$, and the polarization duration was $30 \mathrm{~min}$. The composite film was naturally cooled to room temperature with the voltage kept at $12 \mathrm{kV} / \mathrm{mm}$.

$\mathrm{X}$-ray diffraction (XRD) was employed to analyze the crystal structure and components of the composite films. The microstructure of the composite films was observed by scanning electron microscopy (SEM), and the element distribution of the composite films was analyzed by energydispersive spectroscopy (EDS). A quasi-static $d_{33}$ tester (Institute of Acoustics, Chinese Academic of Science ZJ-3A) was employed to measure the $d_{33}$ value of the composite films. The capacitances $(C)$ and dielectric losses $(\tan \delta)$ were measured using an LCR meter (Tonghui TH2826) at room temperature and a frequency of $1 \mathrm{kHz}$. The relative dielectric constant $\left(\varepsilon_{\mathrm{r}}\right)$ was calculated using Eq. (1):

$$
\varepsilon_{\mathrm{r}}=C \times d /\left(\varepsilon_{0} \times A\right),
$$

where $C$ is the capacitance of the sample, $d$ is the thickness of the sample, $\varepsilon_{0}$ is the permittivity of the vacuum $\left(8.85 \times 10^{-12} \mathrm{~F} / \mathrm{m}\right)$, and $A$ is the effective area of the sample. For each sample, five duplicate tests were conducted at different positions on the samples to minimize the operation error.

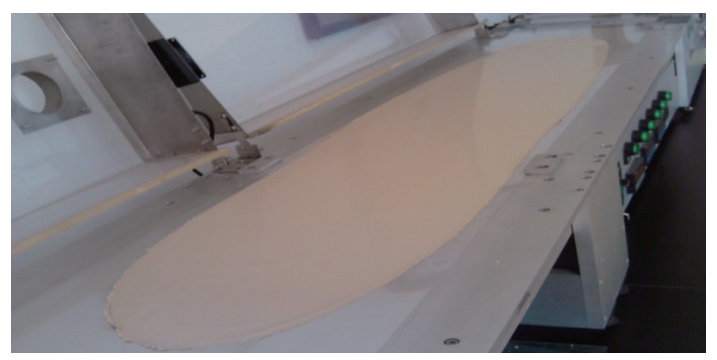

Fig. 1. (Color online) Photograph of the composite film. 


\section{Results and Discussion}

\subsection{Microstructure analysis of the composite films}

Figure 2 shows photographs of the PPF and PPAF with an 85\% volume fraction of PZT. As shown in Fig. 2(a), cracks are clearly observed, which formed during the drying process. The high volume fraction of the PZT in PPF is responsible for the formation of the cracks. The fragile PPF is damaged easily. Compared with the PPF, the PPAF is smooth and flexible, as shown in Fig. 2(b). It can be concluded that the additives considerably improved the flexibility of the PPAF, which enables the broader application of the PPAF under specific operation conditions.

Figure 3 displays SEM images of the top surface of the PPF and PPAF with an $85 \%$ volume fraction of PZT. It can be observed from Fig. 3(a) that the PZT particles are loosely distributed in the PVB matrix, and many microholes exist in the PPF. The microstructure of the PPF is found to be inhomogeneous. Moreover, agglomeration of the PZT particles is noticeable in the microstructure of the PPF as marked using circles in Fig. 3(a). These microdefects may result in microcrack initiation and deteriorate the mechanical properties of the PPF. The microholes
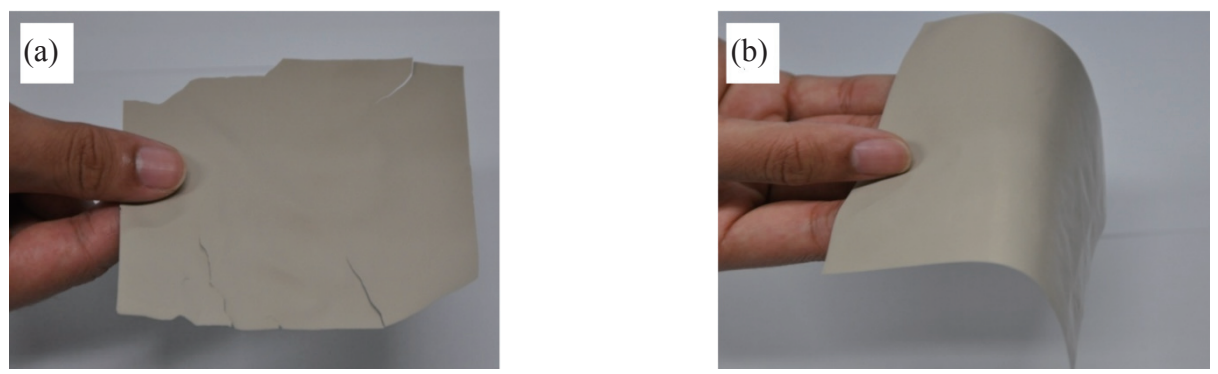

Fig. 2. (Color online) Photographs of the composite films when $\varphi$ (PZT) is $85 \%$ : (a) the PPF after drying and (b) the PPAF after drying.
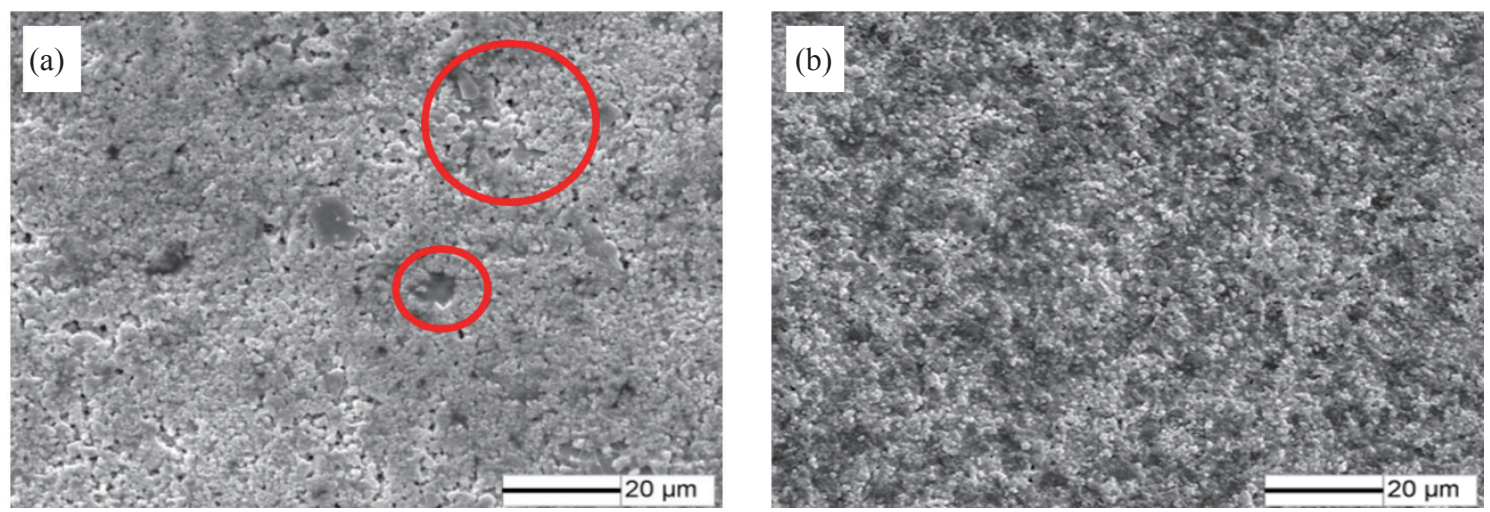

Fig. 3. (Color online) SEM micrographs of the composite films when $\varphi$ (PZT) is $85 \%$ : (a) PPF and (b) PPAF. 
and agglomeration also increase the likelihood that the distribution of the polarization electric field inside the PPF deteriorates during the polarization process. This may lead to the inadequate polarization of the PZT powder and worsen the piezoelectric properties of the PPF. By comparison, it can be observed from Fig. 3(b) that the PZT particles are well dispersed in the PVB matrix, and there are few visible microholes. The microstructure of the PPAF is more homogeneous than that of the PPF. This indicates that the combination of the PZT powder and the PVB matrix is significantly improved as a result of the introduction of the additives. The improved homogeneity and fewer microdefects can also improve the piezoelectric performance of the PPAF.

The EDS results for the composite films with an $85 \%$ volume fraction of PZT are shown in Fig. 4. Figures 4(a) and 4(b) show the top and bottom surfaces of the PPF, respectively. Figures 4(c) and 4(d) show the top and bottom surfaces of the PPAF, respectively. It can be seen from Figs. 4(a) and 4(b) that the Pb content at the bottom surface of the PPF is much higher than that at the top surface. Since $\mathrm{Pb}$ is the main element of PZT, it reveals that the PZT particles were deposited at the bottom surface during the process of tape casting owing to their high density. However, the difference in the $\mathrm{Pb}$ content between the top and bottom surfaces of the PPAF is smaller than that of the PPF, as shown in Fig. 4. This indicates that a uniform distribution of PZT particles is achieved by introducing the additives. The uniform distribution of the PZT particles can greatly enhance the polarization of the PPAF.

Figure 5 shows the XRD patterns of the composite films and their raw materials. It can be observed that the pure PVB powder shows a typical noncrystal structure while the pure PZT powder shows a tetragonal perovskite structure. The crystal structures of the PPF and PPAF are both consistent with that of the pure PZT powder. However, their maximum intensities are clearly

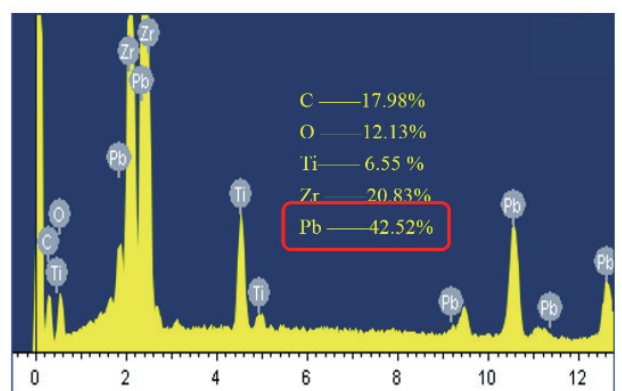

(a)

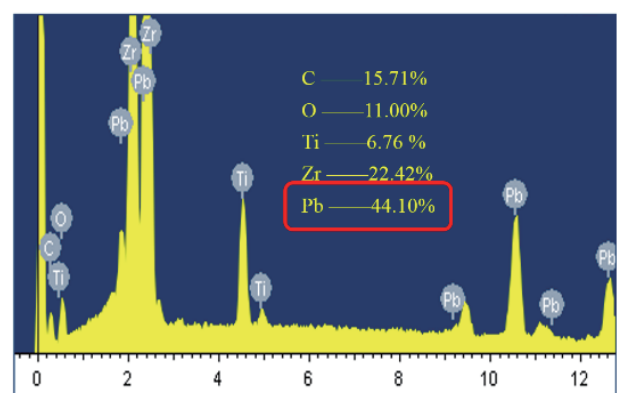

(c)

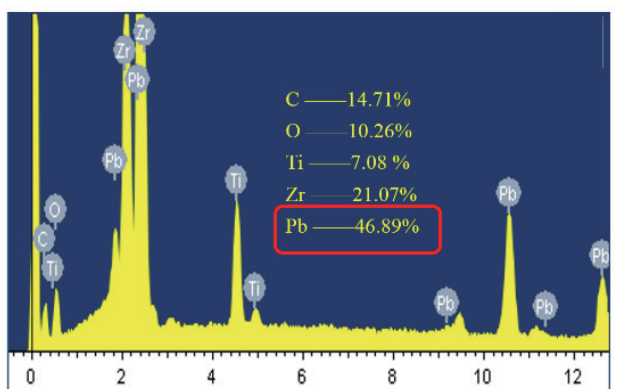

(b)

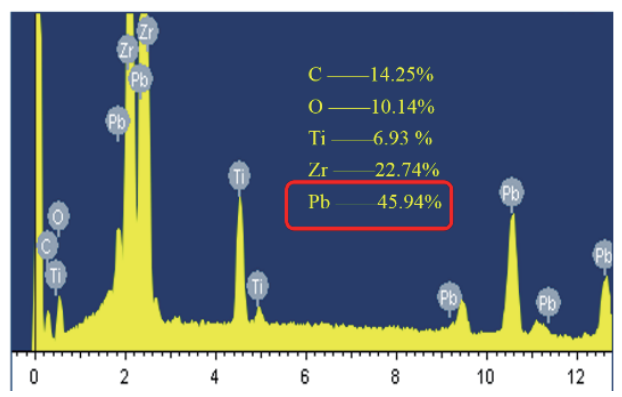

(d)

Fig. 4. (Color online) EDS results of the composite films when $\varphi$ (PZT) is 85\%: (a) top surface of PPF, (b) bottom surface of PPF, (c) top surface of PPAF, and (d) bottom surface of PPAF. 


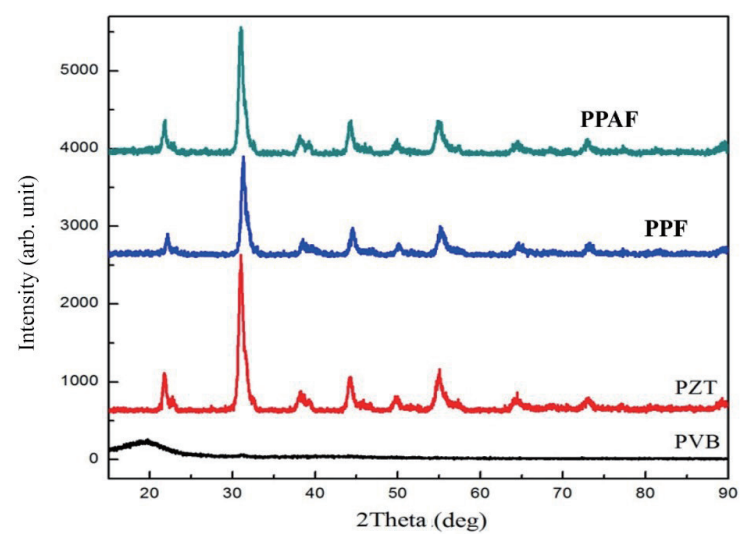

Fig. 5. (Color online) XRD patterns of the composite films and raw materials.

lower owing to the fact that both PPF and PPAF have a lower volume fraction of PZT compared with the pure PZT. In addition, the peak intensity of the PPAF is higher than that of the PPF owing to the fact that the microstructure is dense with few microholes left in the PPAF.

Based on the above analysis, the microstructure of the PPAF is improved considerably compared with that of the PPF. This improvement can enhance the dielectric and piezoelectric properties of the PPAF. A detailed analysis of the dielectric and piezoelectric properties of the PPF and PPAF is demonstrated below.

\subsection{Dielectric properties of the composite films}

Figure 6 demonstrates the relationships between the relative dielectric constants $\left(\varepsilon_{\mathrm{r}}\right)$ and $\varphi(\mathrm{PZT})$ of the PPF and PPAF at room temperature and a frequency of $1 \mathrm{kHz}$. The relationship between the dielectric loss $(\tan \delta)$ and $\varphi(\mathrm{PZT})$ is also shown in Fig. 6. The $\varepsilon_{\mathrm{r}}$ values of the two composite films both increase with increasing $\varphi(\mathrm{PZT})$. This is caused by the fact that the dielectric property of the composite films depends mainly on PZT. When $\varphi(\mathrm{PZT})<60 \%$, the $\varepsilon_{\mathrm{r}}$ values of both PPF and PPAF are almost identical. When $\varphi(\mathrm{PZT})>60 \%$, the $\varepsilon_{\mathrm{r}}$ values of the PPAF are significantly higher than those of the PPF at each of the volume fractions of PZT examined in this study. The main reason is concluded as follows: when $\varphi$ (PZT) $<60 \%$, the PVB matrix is capable of making the PZT particles disperse uniformly, and the effect of the additives on the combination of the PZT particles and the PVB matrix is not significant. However, as $\varphi(\mathrm{PZT})$ increases, the dispersibility of the PZT particles in the matrix decreases, and the additives in such a case play an important role in improving the combination of the PZT particles and the PVB matrix. Consequently, the PPAF exhibits an $\varepsilon_{\mathrm{r}}$ value higher than that of the PPF. In particular, the $\varepsilon_{\mathrm{r}}$ value of the PPAF at $85 \%$ volume fraction of PZT is 93, which is much higher than that of the PZT/PVDF composite film $\left[\varepsilon_{\mathrm{r}}=\right.$ 50 at $\varphi(\mathrm{PZT})=80 \%]^{(25)}$ under the same experimental conditions.

As shown in Fig. 6, the $\tan \delta$ values of the two composite films both increase with increasing $\varphi$ (PZT) because of the energy loss mainly caused by the presence of the PZT particles. However, the $\tan \delta$ value of the PPAF is much lower than that of the PPF at each of the volume fractions of PZT. Moreover, when $\varphi(\mathrm{PZT})$ is $85 \%$, the tan $\delta$ value of the PPAF (0.021) is much lower than that of 


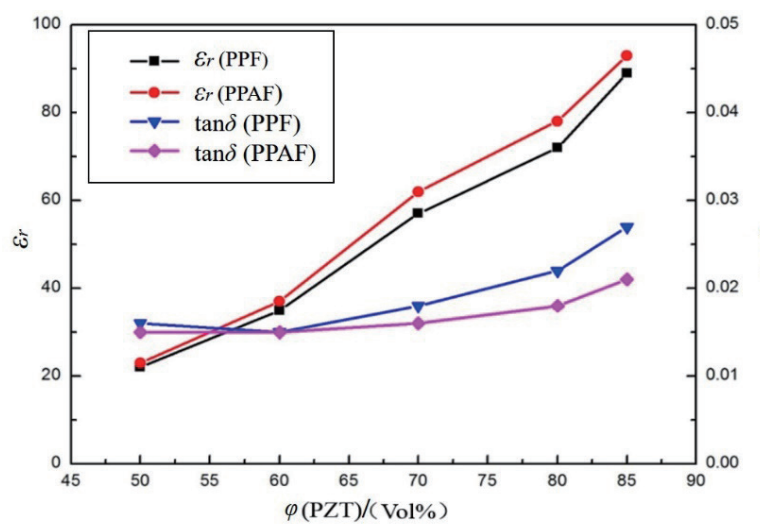

Fig. 6. (Color online) Relationships among $\varepsilon_{\mathrm{r}}, \tan \delta$, and $\varphi(\mathrm{PZT})$ of the composite films.

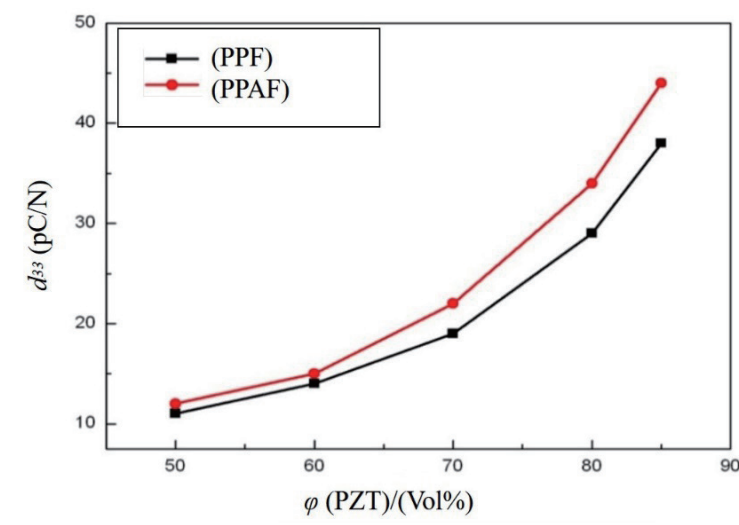

Fig. 7. (Color online) Relationships between $d_{33}$ and $\varphi(\mathrm{PZT})$ of the composite films.

the PZT/PVDF composite film [ $\tan \delta=0.037$, at $\varphi(\mathrm{PZT})=80 \%$ ] reported in Ref. 25 . This further confirms that the uniformity of the composite film microstructure is greatly improved by using additives. The addition of the additives results in fewer microholes and reduces the extra energy loss caused by the microdefects.

\subsection{Piezoelectric properties of the composite films}

Figure 7 shows the variation of the $d_{33}$ value with the volume fraction of PZT for both PPF and PPAF. It can be observed that the $d_{33}$ values of the two composite films both increase as the volume fraction of PZT increases. The monotonic increase is due to the fact that the piezoelectric properties of the composite films depend mainly on PZT. The $d_{33}$ value of the PPAF is consistently higher than that of the PPF for different volume fractions of PZT. At an $85 \%$ volume fraction of PZT, the $d_{33}$ values of the PPF and PPAF are 44 and $38 \mathrm{pC} / \mathrm{N}$, respectively. However, at such a high volume fraction of PZT, the PPF is very fragile as shown in Fig. 2(a). The uniformity of the microstructure of the PPAF is improved by introducing additives. The improvement of the microstructure enhances the piezoelectric property. Compared with the PPF, the distribution of the polarization electric field of the PPAF with fewer microdefects is more homogeneous and results in the sufficient polarization of the PZT powder. Thus, the PPAF exhibits a better piezoelectric property than the PPF.

\section{Conclusions}

A novel large-area and flexible PPAF was fabricated by the tape casting method. By introducing additives (DBP and castor oil), PZT particles disperse more uniformly in the PVB matrix, and microholes and the agglomeration of PZT are reduced. The microstructure of the PPAF is found to be homogeneous.

The dielectric properties of the PPAF are improved owing to the homogeneous microstructure induced by the additives. At an $85 \%$ volume fraction of PZT, the $\varepsilon_{\mathrm{r}}$ value of the PPAF is 93 , which is higher than that of the PPF. The tan $\delta$ value is 0.021 , which is much lower than that of the PPF. 
The homogeneity of the microstructure also improves the distribution of the polarization electric field of the PPAF, producing more sufficient polarization of the PZT powder. Compared with the PPF, the PPAF exhibits a better piezoelectric property $\left(d_{33}=44 \mathrm{pC} / \mathrm{N}\right)$. The proposed PPAF has excellent flexibility and satisfactory dielectric and piezoelectric properties. It provides a viable way of manufacturing flexible sensors for industrial applications. Currently, the application of the PPAF sensors in Lamb-wave-based structure health monitoring for beam structures is ongoing. In addition, the development of hydroacoustic transducers made of the PPAF is also being conducted.

\section{Acknowledgments}

This work is supported by Beijing Natural Science Foundation (3163037). The support is greatly acknowledged.

\section{References}

1 H. Chen, X. Dong, T. Zeng, Z. Zhou, and H. Yang: Ceram. Int. 33 (2007) 1369.

2 J. Z. Tsai, C. J. Chen, W. Y. Chen, J. T. Liu, C. Y. Liao, and Y. M. Hsin: Sens. Actuators, B 139 (2009) 259.

3 N. Jaitanong, A. Chaipanich, and T. Tunkasiri: Ceram. Int. 34 (2008) 793.

4 R. Newnham, D. Skinner, and L. Cross: Mater. Res. Bull. 13 (1978) 525.

5 A. Safari, R. Newnham, L. Cross, and W. Schulze: Ferroelectrics 41 (1982) 197.

6 A. Zak, W. Gan, W. Majid, M. Darroudi, and T. Velayutham: Ceram. Int. 37 (2011) 1653.

7 S. Wang, T. Xu, J. Han, and S. Du: Acta. Mater. Compos. Sinica. 17 (2000) 1.

8 X. Renxin, C. Wen, Z. Jing, L. Yueming, and S. Huajun: J. Wuhan Univ. Technol.-Mater. Sci. Ed. 21 (2006) 84.

9 K. Arlt and M. Wegener: IEEE Trans. Dielectr. Electr. Insul. 17 (2010) 1178.

10 Y. Son, S. Kweon, S. Kim, Y. Kim, T. Hong, and Y. Lee: Integr. Ferroelectr. 88 (2007) 44.

11 Y. Kobayashi, T. Tanase, T. Tabata, T. Miwa, and M. Konno: J. Eur. Ceram. Soc. 28 (2008) 117.

12 A. Holmes-Siedle, P. Wilson, and P. Verrall: Mater. Des. 4 (1983) 910.

13 X. Guan, Y. Zhang, H. Li, and J. Ou: Sens. Actuators, A 194 (2013) 228.

14 X. F. Liu, C. X. Xiong, H. J. Sun, L. J. Dong, and Y. Liu: Mater. Sci. Eng., B 127 (2006) 261.

15 S. Liu, S. Xue, W. Zhang, and J. Zhai: Ceram. Int. 40 (2014) 15633.

16 A. Jain, S. Kumar, M. Kumar, A. Ganesh, and S. Srikanth: Mech. Adv. Mater. Struct. 21 (2014) 181.

17 L. Dong, C. Xiong, H. Quan, and G. Zhao: Scri. Mater. 55 (2006) 835.

18 H. Y. Quan, X. Xie, L. Dong, and C. Xiong: Piezoelectrics and Acoustooptics 3 (2010) 34.

19 X. L. Xie and H. Y. Quan: J. Nanchang Hangkong Univ. (Nat. Sci.) 2 (2012) 014.

20 Y. Zhang: Proc. SEM X Int. Congr. Exposition Exp. Appl. Mech. (2004) pp. 7-10.

21 P. Gimeno, S. Thomas, C. Bousquet, A. Maggio, C. Civade, C. Brenier, and P. Bonnet: J. Chromatogr. B 949 (2014) 99.

22 A. Taylor and J. Harmon: Polym. Degrad. Stab. 41 (1993) 9.

23 W. K. Sakamoto, P. Marin, and D. K. Das-Gupta: Sens. Actuators, A 100 (2002) 165.

24 J. K. Song, W. S. Um, H. S. Lee, M. S. Kang, K. W. Chung, and J. H. Park: J. Eur. Ceram. Soc. 20 (2000) 685 .

25 J. Zhang, W. G. Chen, and C. He: Mater. Mech. Eng. 8 (2012) 007. 